\title{
Neutrinos and Gamma Rays from Photomeson Processes in Gamma Ray Bursts
}

\author{
Armen Atoyan* and Charles D. Dermer ${ }^{\dagger}$ \\ ${ }^{*}$ Centre de Recherche Mathématiques, Université de Montréal, Montréal, Canada H3C 3J7 \\ ${ }^{\dagger}$ Code 7653, Naval Research Laboratory, Washington, DC 20375-5352 USA
}

\begin{abstract}
Acceleration of high-energy hadrons in GRB blast waves will be established if highenergy neutrinos are detected from GRBs. Recent calculations of photomeson neutrino production are reviewed, and new calculations of high-energy neutrinos and the accompanying hadronic cascade radiation are presented. If hadrons are injected in GRB blast waves with an energy corresponding to the measured hard X-ray/soft $\gamma$-ray emission, then only the most powerful bursts at fluence levels $\gtrsim 3 \times 10^{-4} \mathrm{erg} \mathrm{cm}^{-2}$ offer a realistic prospect for detection of $v_{\mu}$. Detection of high-energy neutrinos are likely if GRB blast waves have large baryon loads and Doppler factors $\lesssim 200$. Significant limitations on the hadronic baryon loading and the number of expected neutrinos are imposed by the fluxes from pair-photon cascades initiated in the same processes that produce neutrinos.
\end{abstract}

\section{INTRODUCTION}

In recent work [1], we considered neutrino production for two leading scenarios for the sources that power long-duration GRBs, namely the collapsar [2] and supranova (SA) [3] models. In the collapsar model, the core of a massive star collapses directly to a black hole, and the most important radiation field for photomeson neutrino production is the internal synchrotron radiation field [4]. In the supranova (SA) model, a pulsar nebula synchrotron radiation within an expanding supernova remnant (SNR) shell [5] provides additional external photon target for photomeson interactions (see also Refs. [6, 7]).

We found that the presence of the external field in the SA model can increase the number of detectable neutrinos by an order of magnitude or more over the collapsar model when the Doppler factor $\delta \gtrsim 200$. When $\delta \lesssim 200$, the internal synchrotron field can become effective for photomeson interactions. In our calculations, we assumed that the energy injected in protons is equal to the energy of electrons producing the photon fluence measured at X-ray and $\gamma$-ray energies. In both models, the likelihood of detecting a neutrino from a GRB with a km-scale detector such as IceCube is small except for rare GRBs with fluence $\gtrsim 3 \times 10^{-4} \mathrm{erg} \mathrm{cm}^{-2}$. If GRB blast waves are strongly baryonloaded, however, as required in a GRB model for high-energy cosmic rays [8], then we predict that $100 \mathrm{TeV}-100 \mathrm{PeV}$ neutrinos will be detected several times per year with IceCube when $\delta \lesssim 200$.

Here we summarize our calculations of photomeson neutrino production for the collapsar and SA models, and present new calculations for the hadronic cascade radiation from a GRB. 


\section{MODEL}

The GRB model is adapted from our photo-hadronic model for blazar jets [9], and takes into account the injection of nonthermal protons which lose energy through photomeson interactions. Protons are injected with a number spectrum $\propto E_{p}^{-2}$ at comoving proton energies $E_{p}>\Gamma \mathrm{GeV}$ up to a maximum proton energy determined by the condition that the particle Larmor radius is smaller than both the size scale of the emitting region and the photomeson energy-loss length. Here $\Gamma$ is the Lorentz factor of the blast wave. The observed synchrotron spectral flux in the prompt phase of the burst is parameterized by the expression $F(v) \propto v^{-1}\left(v / v_{b r}\right)^{\alpha}$, where $h v_{b r}=300 \mathrm{keV}, \alpha=-0.5$ above $v_{b r}$ and an exponential cutoff at $10 \mathrm{MeV}$, and $\alpha=0.5$ when $10 \mathrm{keV} \leq h v \leq h v_{b r}$. At lower energies, $\alpha=4 / 3$. The observed total hard X-ray/soft $\gamma$-ray photon fluence $\Phi_{t o t} \cong t_{d u r} \int_{0}^{\infty} d v F(v)$, where $t_{d u r}$ is the characteristic duration of the GRB. We consider a source at redshift $z=1$ and take the hard X-ray/soft $\gamma$-ray fluence $\Phi_{t o t} \gtrsim 3 \times 10^{-5} \mathrm{erg} \mathrm{cm}^{-2}$. Two or three GRBs should occur each month above this fluence level.

A total amount of energy $E^{\prime}=4 \pi d_{L}^{2} \Phi_{t o t} \delta^{-3}(1+z)^{-1}$ is injected in the form of accelerated proton energy into the comoving frame of the GRB blast wave. Here $z$ is the redshift and $d_{L}$ is the luminosity distance. The energy deposited into each of $N_{s p}$ lightcurve pulses (or spikes) is therefore $E_{s p}^{\prime}=E^{\prime} / N_{s p}$ ergs. We assume that all the energy $E_{s p}^{\prime}$ is injected in the first half of the time interval of the pulse (to ensure variability in the GRB light curve), which effectively corresponds to a characteristic variability time scale $t_{v a r}=t_{d u r} / 2 N_{s p}$. The proper width of the radiating region forming the pulse is $\Delta R^{\prime} \cong t_{v a r} c \delta /(1+z)$, from which the energy density of the synchrotron radiation can be determined [9]. We set the GRB prompt duration $t_{d u r}=100 \mathrm{~s}$, and let $N_{s p}=50$, corresponding to $t_{v a r}=1 \mathrm{~s}$. The magnetic field is determined by assuming equipartition between the energy densities of the magnetic field and the electron energy.

For the SA model, we assume the existence of an external radiation field given by the expression $v L_{v} \propto v^{1 / 2} \exp \left(-v / v_{\text {ext }}\right)$, with $h v_{\text {ext }} \approx 1 \mathrm{keV}$ [5]. The intensity of this field is determined by the assumption that the integral power $L_{e x t}=\int_{0}^{\infty} L_{v} \mathrm{~d} v$ is equal to the power of the pulsar wind $L_{p w} \approx\left(10^{53} \mathrm{erg}\right) / t_{\text {delay }}$, assuming that a total of $\approx 10^{53} \mathrm{erg}$ of pulsar rotation energy is radiated during the time $t_{\text {delay }}$ (which is here set equal to 0.1 yr) from the rotating supramassive neutron star before it collapses to a black hole. The energy $h v_{\text {ext }} \simeq 0.1 \mathrm{keV}$ is the characteristic energy of synchrotron radiation emitted by electrons (of the pulsar wind) with Lorentz factors $\gamma_{p w} \sim 3 \times 10^{4}$ in a randomly ordered magnetic field of strength $\approx 10 \mathrm{G}$. The radius $R=0.05 c t$ is determined by assuming that $0.05 c$ is the mean speed of the SNR shell, and that the external photon energy density $\propto L / 2 \pi R^{2}$.

Fig. 1 shows the total $v_{\mu}$ fluences expected from a model GRB with $N_{s p}=50$ pulses. The thin curves show collapsar model results at $\delta=100,200$, and 300, with $\Gamma=\delta$. The expected numbers of $v_{\mu}$ that a km-scale detector such as IceCube would detect are $N_{v}=3.2 \times 10^{-3}, 1.5 \times 10^{-4}$, and $1.9 \times 10^{-5}$, respectively. There is no prospect to detect $v_{\mu}$ from GRBs at these levels. The heavy solid and dashed curves in Fig. 1 give the SA model predictions of $N_{v}=0.009$ for both $\delta=100$ and $\delta=300$. The equipartition magnetic fields are $1.9 \mathrm{kG}$ and $0.25 \mathrm{kG}$, respectively. The external radiation field in the SA model makes the neutrino detection rate insensitive to the value of $\delta$ (as well 


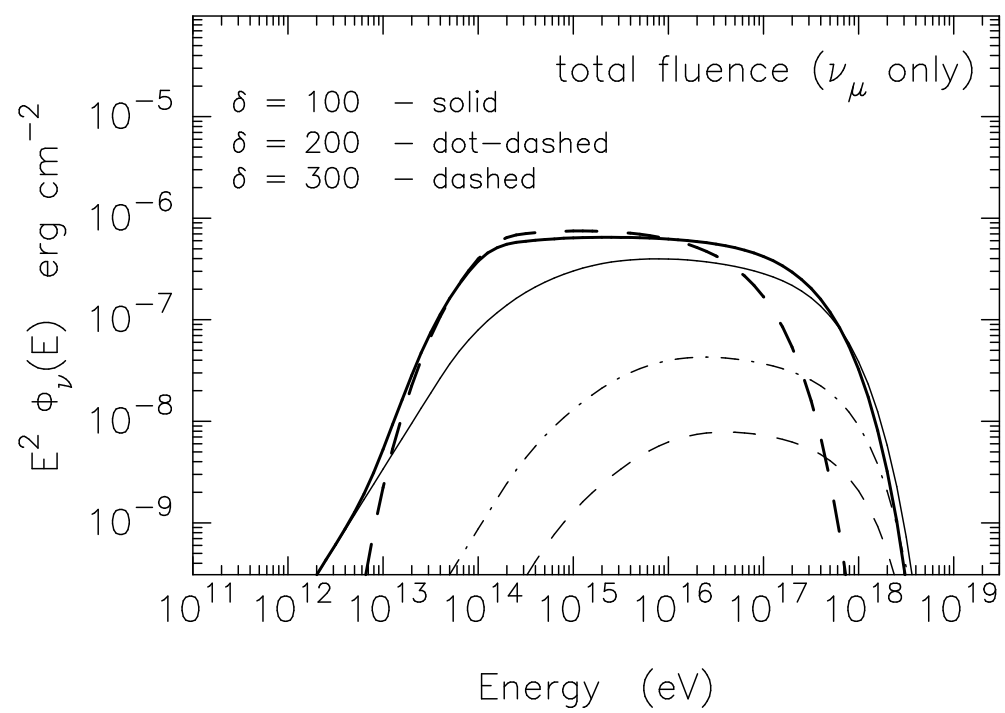

FIGURE 1. Energy fluence of photomeson muon neutrinos $\left(v_{\mu}\right)$ for a model GRB with hard X-ray fluence $\Phi_{t o t}=3 \times 10^{-5} \mathrm{erg} \mathrm{cm}^{-2}$ for different Doppler factors $\delta$. The thin curves show collapsar model results where only the internal synchrotron radiation field provides a source of target photons.

as to $t_{v a r} \gtrsim 0.1 \mathrm{~s}$, as verified by calculations), but there is still little hope that a $\mathrm{km}^{3}$ detector could detect such GRBs. Neutrino production efficiency would improve by at most a factor of 3 in the collapsar model if $t_{v a r} \sim 1 \mathrm{~ms}$ (and $N_{s p}=5 \times 10^{4}$ to provide the same total fluence). Such narrow spikes are, however, then nearly opaque to $\gamma$ rays with energies $\gtrsim 100 \mathrm{MeV}$ [1]. Even in this case, there is little hope to detect neutrinos except from rare GRBs with fluence $\Phi_{t o t} \gtrsim 3 \times 10^{-4} \mathrm{erg} \mathrm{cm}^{-2}$.

Fig. 2 shows new calculations of photomeson neutrino production for a GRB with $\Phi_{t o t}=3 \times 10^{-4} \mathrm{erg} \mathrm{cm}^{-2}$ and $\delta=100$, as well as the accompanying electromagnetic radiation induced by pair-photon cascades from the secondary electrons and $\gamma$ rays from the same photomeson interactions. The total number of $v_{\mu}$ expected with IceCube is $\cong 0.1$. The total fluence of cascade photons shown here is contributed by lepton synchrotron (dot-dashed) and Compton (dashed) emissions. For comparison, the dotted curve shows the primary lepton synchrotron radiation spectrum assumed for the calculations. The level of the fluence of the cascade photons is $\approx 10 \%$ of the primary synchrotron radiation. This means that the maximum allowed baryon loading for these parameters cannot exceed a factor of $\approx 30$ in order not to overproduce the primary synchrotron radiation fluence. This limits the maximum number of $v_{\mu}$ to $\approx 3$ even in the case of large baryon loading for rare, powerful GRBs. These numbers cannot be further increased in the SA model because of the efficient extraction that is already provided by internal photons alone for these parameters.

In conclusion, we predict that at most a few high-energy $v_{\mu}$ can be detected with IceCube even from a very bright GRB at the fluence level $\Phi_{\text {tot }} \gtrsim 3 \times 10^{-4} \mathrm{erg} \mathrm{cm}^{-2}$, and only when the baryon loading is high [1, 8]. This is because the detection of a single $v_{\mu}$ requires a $v_{\mu}$ fluence $\gtrsim 10^{-4} \mathrm{erg} \mathrm{cm}^{-2}$ above $1 \mathrm{TeV}$. Since the energy release in high-energy neutrinos and electromagnetic secondaries is about equal, this energy will 


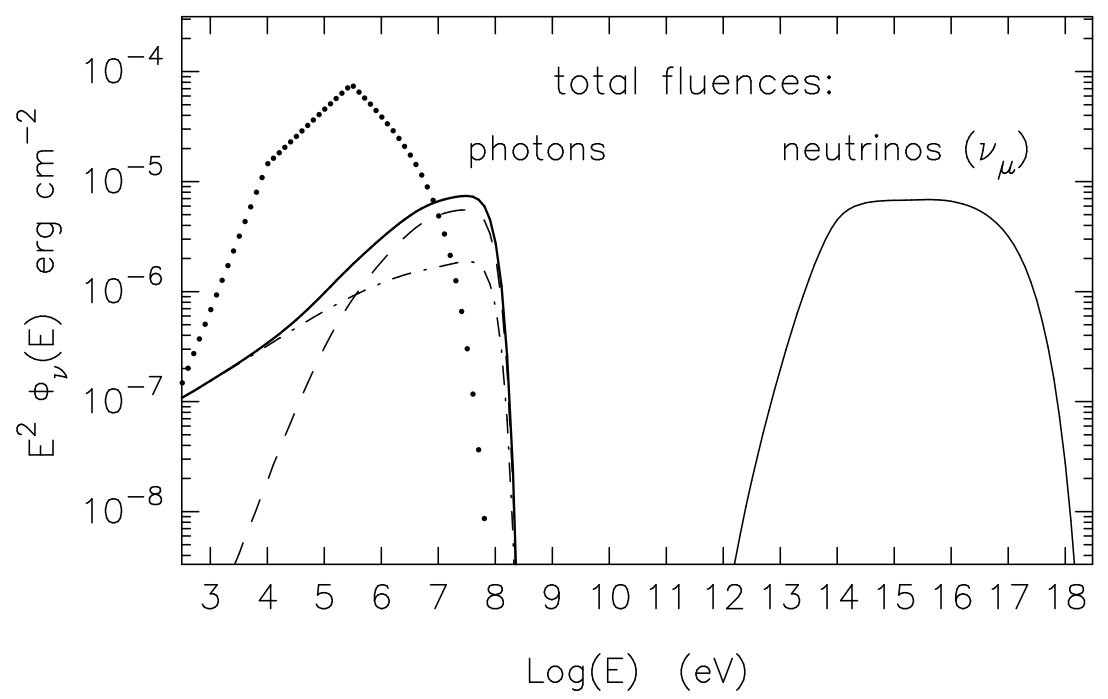

FIGURE 2. Energy fluence of photons and photomeson muon neutrinos for a collapsar-model GRB with hard X-ray fluence $\Phi_{t o t}=3 \times 10^{-4} \mathrm{erg} \mathrm{cm}^{-2}$ and $\delta=100$. The dotted curve shows the fluence of a GRB used for calculations, and the dashed and dot-dashed curves show the Compton and synchrotron contributions to the photon fluence from the electromagnetic cascade initiated by secondaries from photomeson processes, respectively.

be reprocessed in the pair-photon cascade and emerge in the form of observable radiation at hard X-ray/soft $\gamma$-ray energies, and this radiation cannot exceed the measured fluence in this regime. This imposes a robust limit on the maximum number of $v_{\mu}$ even from a GRB with very high baryon loading.

\section{ACKNOWLEDGMENTS}

AA thanks the NRL High Energy Space Environment Branch for support and hospitality during visits. The work of CD is supported by the Office of Naval Research and NASA GLAST science investigation grant DPR \# S-15634-Y.

\section{REFERENCES}

1. C. D. Dermer and A. M. Atoyan, Phys. Rev. Lett. 91, 071102, (2003).

2. C. L. Fryer, S. E. Woosley, and D. H. Hartmann Astrophys. J. 526, 152 (1999).

3. M. Vietri and L. Stella, Astrophys. J. 507, L45 (1998).

4. E. Waxman and J. N. Bahcall, Phys. Rev. Lett. 78, 2292 (1997).

5. A. Königl and J. Granot, Astrophys. J. 574, 134 (2002).

6. Razzaque, S., Meszaros, P., and Waxman, E. Phys. Rev. Lett. 90, 241103 (2003).

7. Guetta, D. \& Granot, J. Phys. Rev. Lett., 90, 201103 (2003).

8. S. D. Wick, C. D. Dermer, and A. Atoyan, Astroparticle Physics, submitted (astro-ph/0310667).

9. A. M. Atoyan and C. D. Dermer, Astrophys. J. 586, 79, (2003). 